\title{
PARA UNA RAZONABLE DEFINICIÓN DE «RAZONABLE»*
}

El concepto de «razonabilidad» o de «razonable» (y de «irrazonabilidad»o «irrazonable») es de una importancia fundamental en la práctica y en la teoría de la argumentación jurídica. La idea de lo razonable en el Derecho aparece a veces como una noción de carácter general, aplicable a cualquier argumentación jurídica, pero a veces también como una noción específica, como un concepto que cobra importancia en cierto tipo de argumentaciones. Un ejemplo de este último uso lo proporciona el art. 14 de la Constitución española («los españoles son iguales ante la ley, sin que pueda prevalecer discriminación alguna por razón de nacimiento, raza, sexo, religión, opinión o cualquier otra condición o circunstancia personal o social»), en cuanto que el mismo plantea un problema de interpretación (sobre qué haya que entender por «igualdad»y por «discriminación») que el Tribunal constitucional (siguiendo, por otro lado, la pauta establecida por el Tribunal europeo de derechos humanos, la Corte suprema estadounidense o el Tribunal constitucional alemán) trata de resolver apelando al concepto de «razonabilidad»: la doctrina, reiterada en numerosos fallos, del tribunal español es que existe discriminación cuando se produce una distinción de trato que carece de una «justificación objetiva y razonable» [cfr., por ejemplo, E. Alonso García, 1983]. Por lo demás, este mismo concepto de razonabilidad o de razonable (en cuanto concepto específico) es designado muchas veces con otras expresiones como «buen padre de familia», «diligencia debida», «trato equitativo», etc. Generalizando más, podría decirse que la noción de razonabilidad es un componente común de lo que suele llamarse «conceptos jurídicos indeterminados»; estos conceptos vendrían a ser el resultado de la aplicación, a campos distintos, de un mismo concepto básico, el de razonabilidad. La afirmación de que lo razonable en el Derecho es una noción de contenido variable [Perelman, 1984] o un valor función [MacCormick, 1984] debe entenderse, pues, tanto en un

\footnotetext{
${ }^{*}$ Este trabajo constituye una primera aproximación a un tema del que espero poder ocuparme con intensidad en los próximos meses. Soy plenamente consciente de sus muchas insuficiencias y lo publico con la intención de recibir críticas y sugerencias de cara a su desarrollo. Agradezco ya desde ahora las observaciones que me han formulado Jesús Ezquerro, Isabel Espejo y Ernesto Garzón Valdés y que sin duda han contribuido a que mi aproximación al tema de lo razonable en el Derecho sea más «razonable» de lo que de otra manera hubiera sido.
} 
sentido histórico o social (lo que haya que considerar como razonable depende de circunstancias temporales y espaciales) como en un sentido lógico (qué haya que entender por razonable en cada caso depende -está en función- del campo a que se aplique la noción).

La importancia práctica del concepto de razonabilidad explica que el mismo ocupe un lugar central en diversas teorías de la argumentación jurídica. Como ejemplos pueden citarse las obras de Luis Recaséns Siches, Chaim Perelman, Neil MacCormick o Aulis Aarnio. Recaséns Siches, por ejemplo, planteó ya desde los años 50 [cfr. Recaséns Siches, 1956 y 1971] la insuficiencia o inadecuación de la lógica tradicional, de la lógica físico-matemática o de lo «racional» para tratar con problemas prácticos como el de la interpretación del Derecho. En su opinión, «la lógica formal (...) no agota ni remotamente la totalidad del logos, de la Razón, sino que es tan sólo una provincia o un sector (...). Aparte de la lógica de lo racional, aparte de la lógica formal de la inferencia, hay otras regiones que pertenecen igualmente al logos, pero que son de índole muy diversa de aquella lógica de lo racional en sentido estricto. Entre esas otras zonas o regiones (...), figura el ámbito del logos de los problemas humanos de conducta práctica, al que yo he llamado logos de lo razonable». [Recaséns Siches, 1971, p. 519]. El hecho de que en los problemas jurídicos, en particular, en los problemas de interpretación, no pueda utilizarse la lógica formal no implicaría, pues, «una fuga de la lógica» [Ibídem, p. 522], sino la utilización de una lógica distinta: la lógica de lo humano o de lo razonable, que es una lógica material, una lógica de los contenidos. Me parece, sin embargo, que el concepto de lo «razonable» (o del «logos de lo razonable») no llega a tener, en la obra de Recaséns Siches, un grado elevado de elaboración teórica. La caracterización que ofrece del mismo gira en torno a una serie de afirmaciones de carácter sumamente vago, como las siguientes: el logos de lo razonable «está limitado, está condicionado y está influido por la realidad concreta del mundo en que opera»; "está impregnado de valoraciones»; "tales valoraciones son concretas»; «las valoraciones constituyen la base o apoyo para la formulación de propósitos»; «la formulación de propósitos o establecimiento de finalidades no sólo se apoya sobre valoraciones sino que además está condicionado por las posibilidades que depare la realidad humana social concreta»; «el logos de lo humano está regido por razones de congruencia o adecuación» entre «la realidad social y los valores», entre «los valores y los fines», etc.; y «está orientado por las enseñanzas sacadas de la experiencia vital e histórica, esto es, individual y social» [Ibídem, pp. 525-6].

En la teoría de la argumentación o nueva retórica de Perelman, la noción de razonable (contrapuesta también aquí a la de racional) juega igualmente un papel central. Lo irrazonable representa para Perelman el límite negativo de cualquier argumentación jurídica $\mathrm{y}$, en general, de cualquier argumentación práctica. Se trata de una noción variable histórica y socialmente y que además remite a una pluralidad de soluciones posibles; dicho de otra manera, una decisión jurídica no puede (no debe) nunca ser irrazonable, pero dos o más decisiones jurídicas pueden 
ser, todas ellas, razonables. Ahora bien, el que se trate de una noción variable no implica que sea también una noción confusa. No lo sería porque, para Perelman, lo razonable tiene un límite que viene marcado por el auditorio; esta última noción, como es sabido, es central en su concepción de la argumentación [cfr., sobre todo, Perelman-Olbrecht Tyteca, 1970]. «Es irrazonable -afirma Perelman- lo que es inadmisible en una comunidad en un momento dado» [Perelman, 1984, p. 15]. «Mientras que, en el Derecho, las ideas de razón y de racionalidad han estado unidas, por una parte, a un modelo divino y, por otra parte, a la lógica y a la técnica eficaz, las de razonable y su opuesta, lo irrazonable, están ligadas a las reacciones del medio social y a su evolución. Mientras que las nociones de «razón» y de «racionalidad» se vinculan a criterios bien conocidos de la tradición filosófica, como las ideas de verdad, de coherencia y de eficacia, lo razonable y lo irrazonable están ligados a un margen de apreciación admisible y a lo que, excediendo de los límites permitidos, parece socialmente inaceptable» [Ibídem, p. 19]. Como lo ha indicado Aarnio [cfr. Aarnio, 1987, 1, p. 222], el concepto de auditorio (de acuerdo del auditorio) que marca el límite de lo aceptable o de lo razonable, adolece, en la obra de Perelman, de una cierta ambigüedad pues, por un lado, se configura como un acuerdo o un consenso ideal y, por otro lado, parece estar ligado a hechos contingentes de carácter social y cultural. Otra dificultad del concepto me parece que estriba en su escasa potencialidad crítica, desde el momento en que Perelman presupone que, ante un mismo caso, pueden tomarse diversas decisiones que (si respetan ciertos límites, que parecen configurarse de manera bastante clara) habría que considerar como aceptables y, por tanto, como igualmente justificables.

En los apartados que siguen de este trabajo me propongo presentar (de manera provisional y esquemática) un análisis del concepto de razonable que se inspira, sobre todo, en trabajos de MacCormick [1984] y de Aarnio [1987,1 y 1987,2]. Entre ambos autores hay un acuerdo de fondo en cuanto a qué haya de entenderse por razonable, pero me parece que cabría afirmar que mientras que MacCormick enfatiza la necesidad de buscar un equilibrio entre exigencias contrapuestas, Aarnio se centra sobre todo en la necesidad de que la decisión logre aceptabilidad o consenso. De todas formas, ambas exigencias están ligadas entre sí, hasta el punto de que mostrar de qué manera lo están constituye, en mi opinión, uno de los puntos centrales que debe resolver un concepto adecuado de lo razonable. El otro punto consiste en elaborar una noción que pueda verse como una reconstrucción de lo razonable tal y como se utiliza en la práctica de la argumentación jurídica pero que, al mismo tiempo, tenga una cierta potencialidad crítica, esto es, pueda utilizarse como un criterio, o un esquema de criterio, que ayude a justificar por qué una determinada interpretación o decisión es preferible (está más justificada) que otra.

2. Para entender qué pueda significar en contextos jurídicos una frase como «X es razonable» hay que empezar por mostrar cuáles son los posibles sujetos de la misma, es decir, de qué entidades puede predicarse 
el carácter de razonable. En principio parecen existir tres posibilidades, en cuanto que $\mathrm{X}$ podría representar: 1) un enunciado jurídico, como una norma, un principio, una definición... o alguna combinación de enunciados de estos tipos; 2) un agente jurídico (un individuo o un grupo), como un legislador, un juez, un tribunal, un funcionario, un abogado, un jurista teórico, etc.; 3) un acto consistente en establecer, interpretar o aplicar enunciados jurídicos. Aquí me ocuparé de los actos consistentes en interpretar y aplicar enunciados jurídicos a casos, a los que denominaré decisiones jurídicas, pues el resultado de dicha actividad consiste en proponer o establecer decisiones. Tales decisiones adoptan, por otro lado, la forma de enunciados lingüísticos, lo que permite distinguir dos tipos de enunciados jurídicos (o, quizás mejor, dos funciones distintas de los enunciados jurídicos): 1) los que se utilizan para decidir casos, y 2) los que son el resultado de la decisión de casos. La definición de «decisión jurídica razonable» que propondré en el próximo apartado puede entenderse que lo es también de este segundo tipo de enunciados jurídicos, pero no de los del primer tipo; es decir, excluyo del campo de la definición las decisiones que consisten en establecer enunciados jurídicos con el propósito de que sean utilizados en el futuro para decidir casos (o, si se quiere precisar más, se excluyen en la medida en que puedan cumplir esta función, pues es posible que un mismo enunciado o conjunto de enunciados jurídicos -por ejemplo, una sentencia- cumplan ambas funciones). Obviamente, las decisiones jurídicas (razonables o irrazonables) son adoptadas por agentes jurídicos, pero esto no quiere decir tampoco que esta última noción pueda reducirse a la primera. O, para ser más exactos, si por «agente jurídico» se entiende el órgano que ha adoptado una determinada decisión, entonces sí que cabría decir que un agente jurídico es razonable si y en la medida en que adopta decisiones jurídicas razonables. Pero por «agente jurídico» cabría también entender el conjunto o la comunidad de individuos o instituciones que adoptan (real o hipotéticamente) decisiones jurídicas, en cuyo caso, la noción de «agente jurídico razonable» (o, quizás mejor, agente racional) no podría derivarse de la de «decisión jurídica razonable», sino que más bien esta segunda presupone la primera.

Otra puntualización a establecer es que se puede hablar de «decisiones jurídicas razonables» en dos sentidos distintos. En un sentido estricto, la razonabilidad puede predicarse únicamente de cierto tipo de decisiones jurídicas: las que no podrían (no deberían) adoptarse siguiendo criterios estrictamente racionales; cuáles sean esos criterios se indicará en el apartado 3. 1. En un sentido amplio, puede decirse que todas las decisiones jurídicas deben ser razonables. Es decir, la razonabilidad operaría como un límite o criterio general que afectaría tanto a las decisiones propiamente razonables como a las estrictamente racionales. Podríamos entonces clasifiicar las decisiones jurídicas en tres grupos: 1) las razonables pero no estrictamente racionales; 2) las razonables y estrictamente racionales; 3) las no razonables (sean o no estrictamente racionales). Las que aquí interesa considerar básicamente son las del primer grupo, esto es, las decisiones jurídicas razonables en sentido estricto, pero, naturalmente, ello implica tomar en consideración también a las del tercer 
grupo, en cuanto que difícilmente podría aclararse la noción de razonable con independencia de la de irrazonable.

Empleo, en fin, la expresión «estrictamente racional» en lugar de «racional» (a secas), para evitar contraponer lo razonable a lo racional, lo que obligaría a su vez a considerar a lo razonable como no racional o como irracional. La idea de que parto es que lo razonable también es racional (tomada esta expresión en un sentido amplio), aunque lo racional pueda no ser razonable: todo lo razonable es racional, pero no todo lo racional es razonable.

3.Una decisión jurídica es razonable en sentido estricto si y sólo si: 1) se toma en situaciones en que no sería aceptable, o no se podría, adoptar una decisión estrictamente racional; 2) logra un equilibrio entre exigencias contrapuestas, pero que necesariamente hay que considerar en la decisión; y 3) es aceptable por la comunidad. Trataré a continuación de aclarar el sentido de los tres requisitos.

3. 1. La noción de inaceptabilidad que aparece en el primer requisito es relativa a la de consenso o acuerdo de la comunidad; de ello me ocuparé con algún detalle en el punto 3.3. Por el momento, me parece interesante señalar ya que mientras que lo razonable apunta a un resultado (que es lo que debe resultar aceptable), la racionalidad estricta es más bien una cuestión procedimental; de ahí que la adopción de decisiones jurídicas siguiendo criterios de estricta racionalidad pueda, en ocasiones, producir resultados inaceptables. Cabría distinguir, quizás, dos tipos distintos de decisiones inaceptables. El primero se define por la existencia de una contradicción entre los resultados que produciría la adopción de la decisión y los objetivos o metas que pretende perseguir el propio sistema jurídico. La adopción en tal caso de una decisión estrictamente racional significaría comportarse de una manera puramente ritualista. En el segundo tipo de decisiones inaceptables, la contradicción se produce entre los resultados de la decisión y valores o fines sociales no contemplados en el sistema jurídico o, directamente, contradictorios con los que incorpora el sistema jurídico. Es obvio que determinar cuándo una decisión lleva a resultados inaceptables plantea más problemas en este segundo caso que en el primero.

Una decisión jurídica es estrictamente racional si y sólo si: 1) Respeta las reglas de la lógica deductiva (lo que Aarnio [1987, 1 y 1987, 2] llama «Racionalidad L»). Por «lógica deductiva» hay que entender aquí la lógica clásica de predicados de primer orden. Esta lógica contiene reglas que tienen poco que ver con las formas naturales de razonar, pero ello no pone en peligro el requisito en cuestión que enuncia sólo una condición necesaria pero no suficiente: para tomar una decisión estrictamente racional hay que no incumplir una regla de la lógica, pero se pueden cumplir todas las reglas de la lógica y tomar decisiones no estrictamente racionales y, desde luego, no razonables. 2) Respeta los principios de la racionalidad práctica (lo que Aarnio [1987, 1 y 1987, 2], inspirándose esencialmente en Alexy [cfr. Alexy, 1978], llama «Racionalidad D»), a saber, los principios de: consistencia, eficiencia, coherencia, generalización y sinceridad; 3 ) Se adopta sin eludir la utilización de alguna 
fuente del Derecho de carácter vinculante. 4) No se adopta sobre la base de criterios éticos, políticos, etc., no previstos específicamente (aunque pudieran estarlo genéricamente) por el ordenamiento jurídico.

Una consecuencia de este requisito de la definición es que la razonabilidad no se plantea en relación con casos claros o rutinarios, sino a propósito tan sólo de los denominados «casos difíciles» (hard cases). En principio podría entenderse que un caso es difícil si, aplicando criterios de racionalidad estricta, resultaría que: a) el caso no tiene solución; b) tiene una solución que resultaría inaceptable; c) tiene más de una solución (incompatibles entre sí). El supuesto a) surge por la imposibilidad de respetar el requisito 4) de la definición de «decisiones jurídicas estrictamente racionales», debido a que se trata realmente de un caso no previsto, o bien de un caso previsto en normas o principios que establecen criterios o pautas de carácter muy general. En el supuesto b), lo que no se puede cumplir es el requisito 3) (para evitar un resultado inaceptable), lo que lleva de nuevo a incumplir 4) (para llegar así a una solución aceptable, razonable). Otro tanto ocurre en el supuesto c), en la medida en que aquí hay que optar por la solución más razonable de las posibles, o bien hay que elaborar una decisión razonable si se entiende que no lo es ninguna de las posibles (utilizando criterios de racionalidad estricta). De esto no se sigue, sin embargo, que las decisiones jurídicas razonables y las estrictamente racionales tengan en común el requisito 1) y 2). Para decirlo con más exactitud, comparten necesariamente el requisito 1), pero pudieran no compartir el 2), en la medida en que el no cumplimiento de 3) implica necesariamente el no cumplimiento de 4) y puede significar también el incumplimiento de alguno de los elementos de 2).

Esto último es, en efecto, lo que parece ocurrir en supuestos en que se toma una decisión sobre la base de una ficción, en forma que no solamente se deja de aplicar una fuente de Derecho de carácter obligatorio, sino que se infringe también, cuando menos, uno de los principios de la racionalidad práctica: el de sinceridad. Como ejemplo puede citarse el caso en que un juez, un tribunal o un jurado considera que el valor de lo robado no superó una cierta cantidad (cuando sabe que no es así y que al afirmarlo está vulnerando las reglas procesales de apreciación de la prueba) para evitar imponer una pena que estima desproporcionadamente dura.

Una decisión jurídica que infringiera las reglas de la lógica deductiva no sería, sin embargo, ni estrictamente racional ni tampoco razonable. Ya se ha dicho que la lógica de predicados de primer orden contiene reglas que se apartan de la forma natural de razonar y cuya utilización, por tanto, podría quizás considerarse como poco «razonable». Pero una decisión razonable puede siempre adoptarse (o, más exactamente, justificarse), sin necesidad de vulnerar ninguna regla de la lógica deductiva aunque, ciertamente, el proceso como tal no consista únicamente (ni quizás esencialmente) en aplicar reglas lógicas, sino más bien en construir premisas utilizando principios interpretativos. Es obvio, por ejemplo, que en el supuesto de ficción que se acaba de señalar la decisión puede tomarse (justificarse) respetando las reglas de la lógica. El esquema lógico 
podría ser el siguiente: las sustracciones de objetos cuyo valor no exceda de la cantidad X deben ser castigadas con la pena Y; A sustrajo objetos cuyo valor no excede de X (el que esta premisa sea falsa, obviamente no afecta a la validez de la deducción lógica); por tanto, A debe ser castigado con la pena $\mathrm{Y}$.

3. 2. En el apartado anterior distinguí tres supuestos en que podían plantearse casos difíciles, esto es, en que los criterios de racionalidad estricta no permitían llegar a una decisión aceptable. En todos estos casos puede hablarse de una contraposición entre valores o principios que deben sopesarse hasta llegar a un equilibrio; dicho equilibrio, naturalmente, no se encuentra siempre (quizás nunca) en el «término medio», puesto que los valores o principios pueden no tener el mismo «peso», de manera que el equilibrio se encontraría entonces en un punto más próximo a un extremo que a otro. $\mathrm{O}$, si se quiere decirlo de otra manera, el término medio está en función de los extremos, pero estos no están ya formados mediante la simple referencia a tales valores o principios, sino que tiene que construirse, en cada caso, a partir de estos materiales. Ocurre a veces que de esta operación resulta una decisión que no es puesta en cuestión por nadie. Tales casos serían ejemplos de decisiones razonables en sentido estricto tal y como se definieron en el apartado 2. (son decisiones razonables, pero no estrictamente racionales), pero en realidad no nos encontramos aquí con casos difíciles en sentido genuino, pues en el fondo lo que hay siempre es un caso con una única decisión aceptable, puesto que es aceptada sin discusión. Los supuestos complicados son aquellos que dan lugar a formas diferentes de encontrar dicho equilibrio, esto es, a soluciones contrapuestas. En tales casos, el concepto de «solución aceptable» se vuelve problemático, pero interesante. Pondré un ejemplo de ello.

En una sentencia reciente [de 27 de octubre de 1987], el Tribunal Constitucional español tuvo que resolver el recurso de inconstitucionalidad interpuesto por el Defensor del Pueblo contra la ley (en realidad dos leyes, una ordinaria y otra orgánica) de objeción de conciencia de 1984 [48/1984, de 26 de diciembre]. Uno de los problemas que aquí se planteaba era el de si debía considerarse o no inconstitucional (por atentar básicamente contra el principio de igualdad del art. 14) el art. 8 ap. 3 de dicha ley que fijaba para el servicio sustitutorio a realizar por los objetores una duración de entre 18 a 24 meses (que debería fijar el Gobierno mediante Real Decreto), mientras que la del servicio militar era de 12 meses. La opinión mayoritaria del tribunal fue la de considerar que la ley no daba lugar a un trato discriminatorio (esto es, que la distinción en cuestión era «razonable y objetiva»), porque los dos supuestos -servicio militar y prestación civil sustitutoria- «no son similares ni cabe equiparar la «penosidad» de uno y otro» (f. j. 5, c). En la argumentación del tribunal jugó un papel importante la tesis de que la objeción de conciencia es un «derecho constitucional autónomo, pero no fundamental» (f. j. 3); el derecho de objeción de conciencia del art. 30, ap. 2 de la Constitución «no es un derecho incondicionado», sino «la exención de un deber general (el servicio militar» (f. j. 5). 
De los tres magistrados que se apartaron del criterio mayoritario (formado en este punto por los nueve magistrados restantes), uno de ellos justificó su voto por entender que «no puede entrar en el ámbito de lo razonable -con toda su relatividad- fijar una duración superior en un $100 \%$, es decir, el doble, para la prestación sustitutoria en relación con la del servicio militar» [voto particular formulado por C. de la Vega, ap. 3]; en su argumentación, el magistrado sostuvo la tesis de que «el derecho a la objeción de conciencia es un derecho constitucionalmente reconocido (...) que participa de la naturaleza de derecho fundamental, per se, es decir, con categoría autónoma, relacionado con el derecho de libertad ideológica» (ap. 1); el servicio sustitutorio y el de armas serían, según esto, «supuestos diferentes en la materialidad de su contenido, pero análogos en cuanto ambos son, en su especie, una manifestación del deber general de servir a España» (ap. 3).

Frente a la cuestión de cuál de las dos soluciones es la razonable (o la «más razonable») cabría responder quizás (utilizando la noción de equilibrio sobre la que gira este segundo requisito de la definición de lo razonable) que lo es la segunda en cuanto que, a diferencia de la primera, busca un punto de equilibrio (que no encuentra en la ley) entre las exigencias del derecho de objeción de conciencia, el deber general de servir a España y el principio de igualdad. Pero esta afirmación no es realmente inobjetable. Si se lee detenidamente la sentencia puede llegarse a la conclusión de que en el criterio mayoritario del tribunal existe también la pretensión de equilibrar esas tres exigencias. Sólo que, como se parte de una interpretación distinta (a la del magistrado discrepante), por un lado, de la relación entre el deber general de defender a España y el derecho de objeción de conciencia $\mathrm{y}$, por otro lado, de la relación de mayor o menor penosidad existente entre la prestación social sustitutoria y el servicio militar, el equilibrio se sitúa aquí en un punto distinto, y de ahí que se entienda que la ley en cuestión no lo rompe.

Naturalmente, si las cosas se dejaran en este punto, el concepto de razonable no serviría para mucho. O, mejor dicho, serviría para demasiado, pues apelando al mismo sería difícil encontrar alguna decisión que no pudiera justificarse. Hay, pues, que seguir planteando el problema de cuál de las dos soluciones (para seguir con el ejemplo anterior) es la razonable, o la más razonable; o, si se quiere, cuál de las dos soluciones logra mejor un equilibrio entre exigencias contrapuestas, pero que no pueden jerarquizarse entre sí de manera que unas excluyan completamente a las otras. Este es, básicamente, el problema para el que debería suministrarnos criterios de solución el tercer requisito de la definición.

3.3. De acuerdo con este tercer requisito habría que decir que, de las dos soluciones en presencia, la más razonable, la que mejor logra un equilibrio, es la que pueda verse como aceptable por la comunidad o, si ambas pudieran serlo en principio, la que suscite un mayor consenso. Ahora bien, de consenso puede hablarse, al menos [cfr. O. Weinberger, 1981], en dos sentidos: como consenso fáctico y como consenso ficticio o ideal. Exploremos ambas posibilidades.

La exigencia de que para que una decisión jurídica pueda ser razonable deba ser aceptada de hecho por una comunidad es una exigencia 
que está llena de sentido. Como tantas veces se ha dicho, la gran ventaja que presentan las decisiones que hemos llamado estrictamente racionales es que, dentro de ciertos límites, resultan previsibles. Lo que asegura dicha previsibilidad es el hecho de que se adoptan siguiendo ciertas reglas procedimentales (los criterios 1) a 4) de la definición de «decisión jurídica estrictamente racional»). Ahora bien, puesto que dichos requisitos no se respetan (es decir, no se respetan íntegramente) en las decisiones razonables, habría que encontrar aquí algún elemento que permitiera, por así decirlo, compensar esta carencia. Y dicho elemento bien pudiera serlo la existencia de un consenso fáctico, es decir, el hecho de que la decisión resulta aceptada por la comunidad. Naturalmente, la aceptación de hecho juega también un papel importante en relación con las decisiones estrictamente racionales, pero cabría decir que su función es, en este caso, negativa: si no se produce aceptación (o un mínimo de aceptación) ello significaría que no se debió adoptar una decisión estrictamente racional.

No parece, sin embargo, que el consenso fáctico pueda considerarse ni como el último ni como el único criterio para elegir entre dos o más decisiones que, en principio, pretendan ser razonables, aunque quizás sí que pudiera pensarse que la no aceptación de hecho (unido a la imposibilidad de dictar una decisión con criterios de estricta racionalidad que resulte aceptable) es una condición suficiente para justificar la adopción de una decisión razonable, en lugar de una estrictamente racional.

Una primera dificultad que plantea el consenso fáctico es que tendría que servir, precisamente, como criterio para resolver casos difíciles, es decir, casos respecto de los cuales no hay un acuerdo claro: una parte de la comunidad, el grupo A, respalda la decisión $\mathrm{X}$, mientras que otra parte, el grupo $\mathrm{B}$, hace lo mismo con $\mathrm{Y}$. La cuestión podría resolverse, naturalmente, utilizando la regla de la mayoría, pero es posible que nos tropezáramos entonces con otro problema, el de qué haya que entender por comunidad. De hecho, en el Derecho operan diversas comunidades o auditorios: el tribunal que tomó la decisión, el conjunto de los tribunales de un país, la doctrina jurídica, los afectados por la decisión, el conjunto de la sociedad, etc. Y no es además nada infrecuente que en los diversos auditorios se produzcan acuerdos mayoritarios, pero de significación contrapuesta; por ejemplo, el criterio más seguido en la práctica es muchas veces mayoritaria o incluso unánimemente rechazado por la doctrina.

De todas formas, la dificultad fundamental del consenso fáctico no radica en esto, sino en el hecho de que en la construcción de los acuerdos juegan siempre un papel los prejuicios sociales, el nivel de información del auditorio, la distinta habilidad argumentativa de quienes sostienen una y otra opinión y, en general, una serie de circunstancias, de factores «espúreos», que hacen que no pueda identificarse sin más «opinión mayoritaria» y «opinión razonable». ¿Debemos excluir a priori la posibilidad de que en ocasiones la opinión razonable -o la más razonable- sea precisamente la opinión de hecho minoritaria?

Si en lugar de a un consenso de tipo fáctico apelamos a uno de tipo ideal o «racional» podemos solucionar quizás estos problemas aunque, 
desde luego, nos vamos a tropezar con algunos otros. Uno es la dificultad de poder utilizar operativamente esta noción, habida cuenta de que la prueba de la razonabilidad (o de la mayor razonabilidad) de una decisión radicaría en un juicio simplemente hipotético: si se cumplieran ciertas condiciones, se produciría acuerdo en torno a una determinada decisión, pero el problema es que quien debe emitir ese juicio hipotético son seres reales, por ejemplo, A y B, y es posible que A pretenda que el acuerdo se produciría en torno a $\mathrm{X}, \mathrm{y}$ $\mathrm{B}$ que en torno a Y. Otra dificultad consiste en la determinación de cuáles sean esas condiciones ideales.

La primera dificultad me parece insalvable, aunque quizás tenga un carácter menos grave de lo que parece a primera vista. Al fin y al cabo, el consenso fáctico también tendría que operar muchas veces hipotéticamente y, después de todo, el razonamiento jurídico es siempre, en un aspecto importante, hipotético en la medida, precisamente, en que es consecuencialista: una manera frecuente de argumentar en Derecho (tan frecuente que se ha podido decir que todo el razonamiento jurídico es en esencia consecuencialista [cfr. $\mathrm{N}$. MacCormick, 1978]) consiste en preferir la solución X a Y, porque X produciría mejores resultados.

Para resolver el segundo problema podría recurrirse a una extensa tradición filosófica que se remonta por lo menos a Hume y a las teorías del espectador imparcial [cfr. J. Muguerza, 1977]. No entraré en ella aquí, sino que me limitaré a suponer que quienes cumplen dichas condiciones ideales serían agentes racionales, suficientemente informados, imparciales y libres, y que estas nociones son suficientemente claras. A partir de aquí puede plantearse el problema de cómo harían tales agentes para ponerse de acuerdo a la hora de establecer principios de justicia de carácter abstracto, y sobre cuáles serían estos. Pero lo que aquí interesa no es exactamente esto, sino contestar más bien a preguntas del tipo: ¿qué pasaría si se situara a dichos agentes frente a un problema concreto como el que suscita la ley de objeción de conciencia?, ¿cómo y en qué punto se alcanzaría el acuerdo?

Yo no creo que una cuestión de este tipo pueda resolverse sencillamente «aplicando» al caso los principios abstractos de justicia que pudieran formularse mediante un consenso ideal y creo, además, que, en general, no es preciso remontarse a esos principios para evaluar decisiones jurídicas (como razonables o irrazonables). A mí me parece que la estrategia a seguir debería consistir en proceder no «de arriba abajo», sino "de abajo arriba» y que pocas veces haría falta llegar muy arriba. Volvamos de nuevo al ejemplo de la ley de objeción de conciencia.

Como hemos visto, la decisión de que la ley es constitucional $\left(\mathrm{D}_{1}\right)$ descansaba en el presupuesto de que el derecho de objeción de conciencia es un derecho autónomo pero no fundamental $\left(\mathrm{P}_{1}\right)$, mientras que la decisión contraria $\left(\mathrm{D}_{2}\right)$ tenía como presupuesto la consideración del derecho de objeción de conciencia como derecho fundamental $\left(\mathrm{P}_{2}\right)$. A partir de aquí, me parece que podría decirse (supuesto -y así me parece de hecho- que ninguna de las dos partes comete en este tramo un «error de argumentación») que si se aceptara $\mathrm{P}_{1}$ el acuerdo (entre seres racionales, etc.) se produciría en relación con $\mathrm{D}_{1}$, pero si se aceptara $\mathrm{P}^{2}$, el acuerdo recaería sobre $\mathrm{D}_{2}$. Con ello naturalmente, lo único que se ha hecho 
es desplazar el problema un paso más atrás. ¿Pero cómo decidir ahora entre $\mathrm{P}_{1}$ y $\mathrm{P}_{2}$ ? Tendríamos que volver a la sentencia para ver cómo se fundamentan, respectivamente, $\mathrm{P}^{1}$ y $\mathrm{P}_{2}$. Si así lo hiciéramos, nos encontraríamos con que tanto el criterio mayoritario del tribunal $\left(D_{1}\right)$ como el del magistrado discrepante $\left(D_{2}\right)$ tienen en cuenta, a la hora de argumentar, respectivamente, en favor de $\mathrm{P}_{1}$ y de $\mathrm{P}_{2}$, una sentencia anterior del propio Tribunal constitucional [15/1982, de 23 de abril]. Cada parte la interpreta de manera distinta, pero el dato relevante aquí es que ambas partes aceptan dicha sentencia, es decir, pretenden seguir (o no apartarse) del criterio sentado allí. Supongamos además que no hay ningún otro argumento en favor de $\mathrm{P}_{1}$ o de $\mathrm{P}_{2}\left(\mathrm{o}\right.$ de $\left.\mathrm{D}_{1} \mathrm{o} \mathrm{D}_{2}\right)$. La cuestión podría plantearse ahora de manera más concreta: Si se acepta el criterio de aquella sentencia (S) y se mantienen las condiciones ideales antes indicadas, ¿cuál de las dos interpretaciones, que llevan respectivamente a $\mathrm{P}_{1}$ y $\mathrm{P}_{2}$, gozaría de un mayor consenso?

Aunque a los efectos de la argumentación no sea importante la respuesta, sino la manera de plantear el problema, yo diría que el acuerdo se produciría en torno a $\mathrm{P}_{2}$. Entre el criterio establecido en la sentencia de 1982 (y recogido en la argumentación del magistrado discrepante), según el cual, la libertad de conciencia constituye una «concreción de la libertad ideológica que nuestra Constitución reconoce en el art. 16» (ap. 1) (el carácter fundamental de este último derecho no ofrece dudas) y $\mathrm{P}_{2}$ (la tesis de que la objeción de conciencia es un derecho fundamental) hay una fuerte y clara conexión, aunque no se trate de una relación de deducibilidad en sentido estricto. Por el contrario, la argumentación de la mayoría del tribunal de que «tampoco de esta sentencia [la de 1982] se infiere que se entendiera que el derecho cuestionado tuviera rango fundamental. Lo que en dicha Sentencia se hizo fue declarar la naturaleza constitucional del derecho a la objeción de conciencia» (f. j. 3), tiene toda la apariencia de un subterfugio que, por otro lado, obliga al tribunal a crear la «extraña» categoría, puesto que sólo albergaría a un miembro, de los «derechos autónomos no fundamentales» [cfr. Capella, 1987] recogida en $\mathrm{P}_{1}$. Si esto es así, habría que decir que la decisión $\mathrm{D}_{1}$ no logra un equilibrio entre las diversas exigencias que se plantean en el caso, sino que sacrifica injustificadamente las derivadas del derecho de objeción de conciencia. $\mathrm{D}_{2}$, es decir declarar en este punto la inconstitucionalidad de la ley, hubiese sido la decisión más razonable.

En resumen, me parece que la estrategia a seguir para hacer operativo el criterio del consenso ideal o ficticio en relación con las decisiones jurídicas razonables tendría que ser la de buscar puntos de acuerdo entre las diversas argumentaciones que tratan de fundamentar decisiones aceptables. Las dificultades aumentarían en la medida en que el acuerdo se produjera sólo en relación con principios o valores de carácter muy abstracto, pero me parece que, afortunadamente, en las cuestiones jurídicas -incluso en aquellas que afectan a las normas jurídicas situadas más arriba en la pirámide normativa- no suele ser este el caso; quien interpreta y aplica el Derecho está siempre vinculado (se siente vinculado) por principios y normas que quizás no despierten su entusiasmo, pero que 
tampoco está dispuesto a poner en cuestión. El control de razonabilidad de las decisiones jurídicas depende, al menos en muy amplia medida, de la búsqueda de estos puntos de acuerdo que, en ocasiones, pueden ser incluso «no queridos».

\section{BIBLIOGRAFÍA}

AARNIO, Aulis, The rational as reasonable. A treatise on legal justification, Reidel, Dordrech/Boston/Lancaster/Tokyo, 1987 (1987, 1).

-Sobre el razonamiento jurídico como razonamiento práctico, X Jornadas de filosofía jurídica y social, Alicante, diciembre, 1987 (1987, 2).

ALEXY, Robert, Theorie der juristischen Argumentation. Die Theorie des rationalen Diskurses als Theorie der juristischen Begründung, Suhrkamp, Frankfurt a.M., 1978.

ALONSO GARCIAA, Enrique, El principio de igualdad del artículo 14 de la Constitución española, en «Revista de Administración Pública», núms. 100-102, enero-diciembre 1983.

CAPELLA, Juan Ramón, GORDILLO, José Luis y ESTÉVEZ, José Antonio, La objeción de conciencia ante el Tribunal Constitucional, en «El País», 30-XI-87.

MACCORMICK, Neil, Legal Reasoning and Legal Theory, Clarendon Press, Oxford, 1978.

-On reasonableness, en Les notions a contenu variable en Droit, vol. dirigido por Ch. Perelman y R. Vander Elst, E. Bruylant, Bruselas, 1984.

MUGUERZA, Javier, La razón sin esperanza. (Siete trabajos y un problema de ética), Taurus, Madrid, 1977.

PERELMAN, Chaim, Le raisonnable el le déraisonnable en droit, en el libro del mismo título, L. G. L. J., París, 1984.

PERELMAN, Chaim y OLBRECHT TYTECA, L, La nouvelle rhétorique, Traité de l'argumentation, Université libre de Bruxelles, Bruselas, 1970.

México, 1956.

RECASENS SICHES, Luis, Nueva filosofía de la interpretación del Derecho, Dianoia,

-Experiencia jurídica, naturaleza de la cosa y lógica «razonada», Dianoia, Mexico, 1971.

Sentencia núm. 160/1987, de 27 de octubre, del Tribunal Constitucional, en B. O. E. núm. 271. Suplemento (12-XI-1987).

WEINBERGER, Ota, Die Rolle des Konsenses in der Wissenschaft, im Recht und in der Politik, en «Rechtstheorie», Beiheft 2, 1981. 